\title{
Fuzzy Availability Assessment for a Repairable Multistate Series-Parallel System
}

\author{
Linmin Hu, ${ }^{1}$ Dequan Yue, ${ }^{1,2}$ and Ruiling Tian' \\ ${ }^{1}$ College of Science, Yanshan University, Qinhuangdao 066004, China \\ ${ }^{2}$ School of Economics and Management, Yanshan University, Qinhuangdao 066004, China \\ Correspondence should be addressed to Dequan Yue; ydq@ysu.edu.cn
}

Received 27 January 2015; Revised 12 April 2015; Accepted 15 April 2015

Academic Editor: Victor S. Kozyakin

Copyright (c) 2015 Linmin Hu et al. This is an open access article distributed under the Creative Commons Attribution License, which permits unrestricted use, distribution, and reproduction in any medium, provided the original work is properly cited.

\begin{abstract}
This paper considers a repairable multistate series-parallel system (RMSSPS) with fuzzy parameters. It is assumed that the system components are independent, and their state transition rates and performance rates are fuzzy values. The fuzzy universal generating function technique is adopted to determine fuzzy state probability and fuzzy performance rate of the system. On the basis of $\alpha$-cut approach and the extension principle, parametric programming technique is employed to obtain the $\alpha$-cuts of some indices for the system. The system fuzzy availability is defined as the ability of the system to satisfy fuzzy consumer demand. A special assessment approach is developed for evaluating the fuzzy steady-state availability of the system with the fuzzy demand. A flow transmission system with three components is presented to demonstrate the validity of the proposed method.
\end{abstract}

\section{Introduction}

The study of repairable systems is an important topic in engineering systems. System availability is very good evaluation for performance of repairable systems and occupies an increasingly important issue in power plants, manufacturing systems, industrial systems, and transportation systems, and so forth.

In the real-world problems, many repairable systems are designed to perform their intended tasks in a given environment. One type of these repairable systems is repairable multistate system (RMSS). The RMSS is able to perform its task with various distinguished levels of efficiency usually referred to as performance rates. Since the number of RMSS states increases very rapidly with the increase in the number of its components, the universal generating function (UGF) technique was introduced and proved to be efficient in evaluating the reliability of the multistate systems [1-4].

The repairable multistate series-parallel system (RMSSPS) model is frequently used in practice and has been extensively studied for many years. The conventional study for the RMSSPS considers the assumptions of the exact state transition rate and performance rate for each system component. However, in many engineering applications it is very difficult to obtain accurate and sufficient data to estimate the precise values of the state transition rate and performance rate for each system component. For this reason, the concept of fuzzy reliability has been introduced and developed by several authors [5-9].

Fuzzy set theory proposed by Zadeh [10] is a very good approach to deal with fuzzy uncertainty and has gained successful applications in various fields. It provides useful tools to investigate and analyze imprecision phenomena in queuing systems [11], rock engineering classification systems [12], transport systems [13], manufacturing systems [14], supply chain problems [15], and various optimization problems [1618]. Wong and Lai [19] provided a survey of applications of the fuzzy set theory technique in production and operations management and pointed out that nearly every application is potentially able to realize some of the benefits of fuzzy set theory. Furthermore, fuzzy set theory also provides useful methodology to analyze the reliability in uncertain systems. It can deal with the problem of lacking of inaccuracy or fluctuation data for system components in reliability analysis of some realistic engineering systems. Thus, it is necessary to introduce fuzzy set theory into the reliability theory to deal with reliability of the system with uncertain parameters. The theory of fuzzy reliability has been developed on the basis of 
fuzzy set theory. Many research works on the application of fuzzy set theory to problems in reliability or availability of systems were presented in [20-23], and a systemic review on fuzzy reliability of systems with binary-state was provided by Cai [24].

Recently, fuzzy reliability research has focused on reliability evaluation of fuzzy multistate systems. Ding et al. [25, 26] proposed firstly the concept of fuzzy multistate system and assessed the fuzzy reliability of fuzzy multistate systems with fuzzy demand using fuzzy UGF method. Liu et al. $[27,28]$ investigated the dynamic fuzzy state probabilities, fuzzy performance rates, and fuzzy availability for fuzzy unrepairable multistate elements and fuzzy unrepairable multistate system according to the parametric programming technique and the extension principle. Bamrungsetthapong and Pongpullponsak [29] discussed fuzzy confidence interval for the fuzzy reliability of a RMSSPS with a fuzzy failure rate and a fuzzy repair rate and considered the performance of fuzzy confidence interval based on the coverage probability and the expected length.

Steady-state availability of a repairable system, as a system performance measure, is the probability that the system is performing satisfactorily over a reasonable period of time [30]. The RMSS availability is defined as the ability of the system to satisfy consumer demand, which is equal to the sum of the probabilities of occurrence of system states in which the system performance rates satisfy consumer demand. Comparable work on the steady-state availability assessment for the RMSSPS with fuzzy state transition rate and fuzzy performance rate is rarely found in the literature. This motivates us to develop the fuzzy steady-state availability assessment for a RMSSPS with fuzzy parameters. According to [4], in this paper, we assumed that the fuzzy performance rate of each multistate parallel subsystem is the sum of the fuzzy performance rates of its all components, and the fuzzy performance rate of the entire RMSSPS is the minimum of the fuzzy performance rates of all parallel subsystems. The purpose of this study is to utilize the $\alpha$-cut approach, the extension principle, and parametric programming technique to determine the fuzzy state probability and fuzzy performance rate of the RMSSPS and to evaluate the fuzzy steadystate availability of the system with the fuzzy consumer demand.

The rest of the paper is organized as follows. Section 2 introduces the description of the system considered here and analyzes the fuzzy state probability of multistate component. The fuzzy state probabilities and fuzzy performance rates of the repairable parallel subsystem and the RMSSPS are presented in Sections 3 and 4, respectively. The fuzzy availability assessment method for the RMSSPS is given in Section 5 . An illustrative example is presented in Section 6. Finally, Section 7 gives conclusions.

\section{RMSSPS with Fuzzy Parameters}

The RMSSPS we considered here is composed of $n$ subsystems connected in series, and each subsystem $i$ consists of $m_{i}$ components in parallel, $i=1,2, \ldots, n$. The structure of a RMSSPS is shown in Figure 1.

Each component $j$ of subsystem $i$ has $M_{i j}+1$ different states corresponding to the fuzzy performance rates which can be represented by ordered fuzzy values $\widetilde{\mathbf{g}}_{i j}=$ $\left(\widetilde{g}_{i j, 0}, \widetilde{g}_{i j, 1}, \ldots, \widetilde{g}_{i j, M_{i j}}\right)$, and $\widetilde{g}_{i j, k}, k=0,1, \ldots, M_{i j}$, is arbitrary fuzzy number. It is assumed that transitions for each component can only occur between adjacent states, and the state transition diagram is shown in Figure 2. The transition rate from state $k$ to state $k-1$ is presented as the fuzzy value $\tilde{\lambda}_{i j}(k, k-1)$, and the transition rate from state $k-1$ to state $k$ is presented as the fuzzy value $\widetilde{\mu}_{i j}(k-1, k), k=1,2, \ldots, M_{i j}$, and $\widetilde{\lambda}_{i j}(k, k-1)$ and $\widetilde{\mu}_{i j}(k-1, k)$ are arbitrary fuzzy numbers.

With the fuzzy transition rate, the state probability of the component $j$ of subsystem $i$ for the steady state is also a fuzzy value denoted as $\widetilde{p}_{i j, l_{j}}, l_{j}=0,1, \ldots, M_{i j}$; let $\widetilde{\mathbf{p}}_{i j}=$ $\left(\widetilde{p}_{i j, 0}, \widetilde{p}_{i j, 1}, \ldots, \widetilde{p}_{i j, M_{i j}}\right)$.

The fuzzy transition rate matrix for the component $j$ of subsystem $i$ is given as

$$
\begin{aligned}
& \tilde{\Delta}_{i j} \\
& =\left(\begin{array}{ccccccc}
-\tilde{\mu}_{i j}(0,1) & \tilde{\mu}_{i j}(0,1) & 0 & \cdots & 0 & 0 & 0 \\
\tilde{\lambda}_{i j}(1,0) & -\left(\tilde{\mu}_{i j}(1,2)+\tilde{\lambda}_{i j}(1,0)\right) & \tilde{\mu}_{i j}(1,2) & \cdots & 0 & 0 & 0 \\
\vdots & \vdots & \vdots & & \vdots & \vdots & \vdots \\
0 & 0 & 0 & \cdots & \tilde{\lambda}_{i j}\left(M_{i j}-1, M_{i j}-2\right) & -\left(\tilde{\mu}_{i j}\left(M_{i j}-1, M_{i j}\right)+\tilde{\lambda}_{i j}\left(M_{i j}-1, M_{i j}-2\right)\right) & \tilde{\mu}_{i j}\left(M_{i j}-1, M_{i j}\right) \\
0 & 0 & 0 & \cdots & 0 & \tilde{\lambda}_{i j}\left(M_{i j}, M_{i j}-1\right) & -\tilde{\lambda}_{i j}\left(M_{i j}, M_{i j}-1\right)
\end{array}\right) .
\end{aligned}
$$

Based on the state transition diagram (Figure 2) and the fuzzy transition rate matrix, the balance equations for steady state of the component $j$ of subsystem $i$ are given by

$$
\begin{aligned}
& \tilde{\mu}_{i j}(0,1) \widetilde{p}_{i j, 0}=\widetilde{\lambda}_{i j}(1,0) \widetilde{p}_{i j, 1}, \\
& \left(\widetilde{\lambda}_{i j}\left(l_{j}, l_{j}-1\right)+\widetilde{\mu}_{i j}\left(l_{j}, l_{j}+1\right)\right) \widetilde{p}_{i j, l_{j}}
\end{aligned}
$$

$$
\begin{aligned}
=\tilde{\lambda}_{i j}\left(l_{j}+1, l_{j}\right) \tilde{p}_{i j, l_{j}+1}+\widetilde{\mu}_{i j}\left(l_{j}-1, l_{j}\right) \tilde{p}_{i j, l_{j}-1}, & \\
& l_{j}=1,2, \ldots, M_{i j}-1,
\end{aligned}
$$$$
\tilde{\lambda}_{i j}\left(M_{i j}, M_{i j}-1\right) \tilde{p}_{i j, M_{i j}}=\tilde{\mu}_{i j}\left(M_{i j}-1, M_{i j}\right) \tilde{p}_{i j, M_{i j}-1} .
$$ 


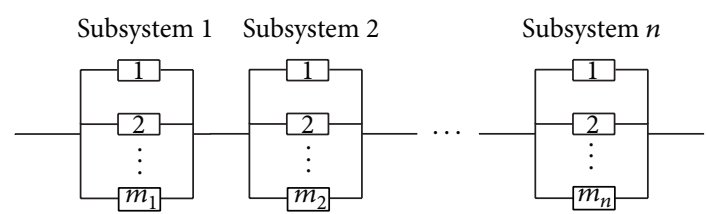

FIGURE 1: General structure of a RMSSPS.

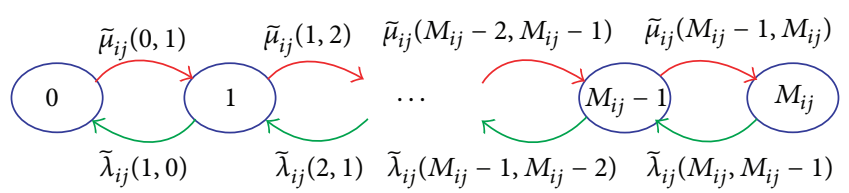

FIgURE 2: The state-transition diagram for the component $j$ of subsystem $i$.

Equations (2) are Chapman-Kolmogorov equations with fuzzy transition rates for steady state. They state that the rate of flow into state $l_{j}$ equals the rate of flow out of state $l_{j}$ in the steady state, $l_{j}=0,1, \ldots, M_{i j}$. Taking the relation $\sum_{l_{j}=0}^{M_{i j}} \widetilde{p}_{i j, l_{j}}=$ 1 into account, we can obtain the state probability $\widetilde{p}_{i j, l_{j}}$ as function of $\tilde{\lambda}_{i j}(k, k-1)$ and $\tilde{\mu}_{i j}(k-1, k), k=1,2, \ldots, M_{i j}$; that is

$$
\begin{aligned}
\tilde{p}_{i j, l_{j}} & =\widetilde{p}_{i j, l_{j}}\left(\widetilde{\lambda}_{i j}(k, k-1), \widetilde{\mu}_{i j}(k-1, k) ;\right. \\
k & \left.=1,2, \ldots, M_{i j}\right) .
\end{aligned}
$$

According to the state probability distribution of the conventional multistate component, we have

$$
\begin{array}{r}
\tilde{p}_{i j, l_{j}} \\
=\frac{\tilde{\mu}_{i j}\left(l_{j}-1, l_{j}\right) \tilde{\mu}_{i j}\left(l_{j}-2, l_{j}-1\right) \cdots \widetilde{\mu}_{i j}(0,1)}{\widetilde{\lambda}_{i j}\left(l_{j}, l_{j}-1\right) \tilde{\lambda}_{i j}\left(l_{j}-1, l_{j}-2\right) \cdots \widetilde{\lambda}_{i j}(1,0)} \widetilde{p}_{i j, 0}, \\
l_{j}=1, \ldots, M_{i j},
\end{array}
$$

where $\widetilde{p}_{i j, 0}=1 /\left(1+\sum_{k=1}^{M_{i j}}\left(\widetilde{\mu}_{i j}(0,1) \widetilde{\mu}_{i j}(1,2) \cdots \widetilde{\mu}_{i j}(k-1, k) /\right.\right.$ $\left.\left.\tilde{\lambda}_{i j}(1,0) \tilde{\lambda}_{i j}(2,1) \cdots \widetilde{\lambda}_{i j}(k, k-1)\right)\right)$.

Let $\eta_{\tilde{\lambda}_{i j}}\left(\lambda_{i j}(k, k-1)\right)$ and $\eta_{\tilde{\mu}_{i j}}\left(\mu_{i j}(k-1, k)\right)$ denote the membership functions of $\tilde{\lambda}_{i j}(k, k-1)$ and $\tilde{\mu}_{i j}(k-1, k)$, respectively. Definitions for the $\alpha$-cuts of $\widetilde{\lambda}_{i j}(k, k-1)$ and $\tilde{\mu}_{i j}(k-1, k)$ as crisp intervals are as follows:

$$
\begin{gathered}
\tilde{\lambda}_{i j}(k, k-1)_{\alpha}=\left[\operatorname { m i n } _ { \lambda _ { i j } ( k , k - 1 ) \in \Lambda _ { i j } ( k , k - 1 ) } \left\{\lambda_{i j}(k, k-1) \mid\right.\right. \\
\left.\eta_{\tilde{\lambda}_{i j}}\left(\lambda_{i j}(k, k-1)\right) \geq \alpha\right\}, \\
\lim _{i j}(k, k-1) \in \Lambda_{i j}(k, k-1) \\
\geq \alpha\}]=\left[\left(\lambda_{i j}(k, k-1)\right)_{\alpha}^{L},\left(\lambda_{i j}(k, k-1) \mid \eta_{\tilde{\lambda}_{i j}}\left(\lambda_{i j}(k, k-1)\right)\right.\right. \\
\alpha
\end{gathered}
$$

$$
\begin{gathered}
\widetilde{\mu}_{i j}(k-1, k)_{\alpha}=\left[\operatorname { m i n } _ { \mu _ { i j } ( k - 1 , k ) \in N _ { i j } ( k - 1 , k ) } \left\{\mu_{i j}(k-1, k) \mid\right.\right. \\
\left.\eta_{\widetilde{\mu}_{i j}}\left(\mu_{i j}(k-1, k)\right) \geq \alpha\right\}, \\
\max _{i j}(k-1, k) \in N_{i j}(k-1, k) \\
\geq \alpha\}]=\left[\left(\mu_{i j}(k-1, k) \mid \eta_{\widetilde{\mu}_{i j}}\left(\mu_{i j}(k-1, k)\right)\right.\right. \\
\left.\geq k))_{\alpha}^{L},\left(\mu_{i j}(k-1, k)\right)_{\alpha}^{U}\right],
\end{gathered}
$$

where $\Lambda_{i j}(k, k-1)$ and $N_{i j}(k-1, k)$ are the crisp universal sets of the transition rate from state $k$ to state $k-1$ and the transition rate from state $k-1$ to state $k$ for the component $j$ of subsystem $i$.

According to Zadeh extension principle [10,31], the $\alpha$-cut of the fuzzy state probability $\tilde{p}_{i j, l_{j}}=\widetilde{p}_{i j, l_{j}}\left(\widetilde{\lambda}_{i j}(k, k-1), \tilde{\mu}_{i j}(k-\right.$ $\left.1, k) ; k=1, \ldots, M_{i j}\right)$ can be obtained as

$$
\begin{aligned}
& \left(\tilde{p}_{i j, l_{j}}\right)_{\alpha}=\left[\operatorname { m i n } _ { \Psi } \left\{p_{i j, l_{j}} \mid \eta_{\tilde{\lambda}_{i j}}\left(\lambda_{i j}(k, k-1)\right)\right.\right. \\
& \left.\geq \alpha, \eta_{\widetilde{\mu}_{i j}}\left(\mu_{i j}(k-1, k)\right) \geq \alpha\right\}, \\
& \max _{\Psi}\left\{p_{i j, l_{j}} \mid \eta_{\tilde{\lambda}_{i j}}\left(\lambda_{i j}(k, k-1)\right)\right. \\
& \left.\left.\geq \alpha, \eta_{\widetilde{\mu}_{i j}}\left(\mu_{i j}(k-1, k)\right) \geq \alpha\right\}\right]=\left[\left(p_{i j, l_{j}}\right)_{\alpha}^{L},\right. \\
& \left.\left(p_{i j, l_{j}}\right)_{\alpha}^{U}\right],
\end{aligned}
$$

where $\Psi=\left\{\lambda_{i j}(k, k-1) \in \Lambda_{i j}(k, k-1), \mu_{i j}(k-1, k) \in N_{i j}(k-\right.$ $\left.1, k) ; k=1, \ldots, M_{i j}\right\}$. Using the parametric programming technique $[27,28,32,33]$, the lower and upper bounds of the $\alpha$-cut of $\widetilde{p}_{i j, l_{j}}$ can be obtained as

$$
\begin{aligned}
\left(p_{i j, l_{j}}\right)_{\alpha}^{L}=\min & p_{i j, l_{j}}, \quad(0 \leq \alpha \leq 1) \\
& \text { s.t. } \quad \lambda_{i j}(k, k-1)_{\alpha}^{L} \leq \lambda_{i j}(k, k-1) \\
& \leq \lambda_{i j}(k, k-1)_{\alpha}^{U} \\
& \mu_{i j}(k-1, k)_{\alpha}^{L} \leq \mu_{i j}(k-1, k) \\
& \leq \mu_{i j}(k-1, k)_{\alpha}^{U}, \quad k=1,2, \ldots, M_{i j}, \\
& \\
\left(p_{i j, l_{j}}\right)_{\alpha}^{U}=\max & p_{i j, l_{j}}, \quad(0 \leq \alpha \leq 1) \\
& \lambda_{i j}(k, k-1)_{\alpha}^{L} \leq \lambda_{i j}(k, k-1) \\
& \leq \lambda_{i j}(k, k-1)_{\alpha}^{U}
\end{aligned}
$$




$$
\begin{aligned}
& \mu_{i j}(k-1, k)_{\alpha}^{L} \leq \mu_{i j}(k-1, k) \\
& \leq \mu_{i j}(k-1, k)_{\alpha}^{U} \\
& \quad k=1,2, \ldots, M_{i j} .
\end{aligned}
$$

\section{Fuzzy Performance Rate and Fuzzy State Probability of the Parallel Subsystem}

According to the following fuzzy UGF of the component $j$ of subsystem $i$

$$
\begin{aligned}
\tilde{u}_{i j}(z) & =\widetilde{p}_{i j, 0} \cdot z^{\widetilde{g}_{i j, 0}}+\widetilde{p}_{i j, 1} \cdot z^{\widetilde{g}_{i j, 1}}+\cdots+\widetilde{p}_{i j, M_{i j}} \cdot z^{\widetilde{g}_{i j, M_{i j}}} \\
& =\sum_{l_{j}=0}^{M_{i j}} \widetilde{p}_{i j, l_{j}} \cdot z^{\widetilde{g}_{i j, l_{j}}}
\end{aligned}
$$

where $\widetilde{g}_{i j, l_{j}}$ is the fuzzy performance rate of the component $j$ of subsystem $i$ in its state $l_{j}$ and $\widetilde{p}_{i j, l_{j}}$ is the fuzzy state probability associated with the state $l_{j}, l_{j}=0,1, \ldots, M_{i j}$.

Using fuzzy UGF technique and fuzzy composition operator $\widetilde{\Theta}_{\phi_{P}}$ [25], we have the fuzzy UGF of subsystem $i$

$\tilde{u}_{i}(z)$

$$
\begin{aligned}
& =\widetilde{\Theta}_{\phi_{P}}\left(\sum_{l_{1}=0}^{M_{i 1}} \tilde{p}_{i 1, l_{1}} \cdot z^{\tilde{g}_{i 1, l_{1}}}, \ldots, \sum_{l_{m_{i}}=0}^{M_{i m_{i}}} \widetilde{p}_{i m_{i}, l_{m_{i}}} \cdot z^{\widetilde{g}_{i m_{i}, l_{m_{i}}}}\right) \\
& =\sum_{l_{1}=0}^{M_{i 1}} \sum_{l_{2}=0}^{M_{i 2}} \cdots \sum_{l_{m_{i}}=0}^{M_{i m_{i}}}\left(\prod_{j=1}^{m_{i}} \widetilde{p}_{i j, l_{j}} \cdot z^{\sum_{j=1}^{m_{i}} \tilde{g}_{i j, l_{j}}}\right) \\
& =\sum_{l_{1}=0}^{M_{i 1}} \sum_{l_{2}=0}^{M_{i 2}} \cdots \sum_{l_{m_{i}}=0}^{M_{i m_{i}}}\left(\tilde{p}_{i, r_{i}} \cdot z^{\widetilde{g}_{i, r_{i}}}\right)=\sum_{r_{i}=0}^{M_{i}} \tilde{p}_{i, r_{i}} \cdot z^{\widetilde{g}_{i, r_{i}}}
\end{aligned}
$$

where $\widetilde{g}_{i, r_{i}}=\sum_{j=1}^{m_{i}} \widetilde{g}_{i j, l_{j}}$ is the fuzzy performance rate of the parallel subsystem $i$ in its state $r_{i}$ and $\widetilde{p}_{i, r_{i}}=\prod_{j=1}^{m_{i}} \widetilde{p}_{i j, l_{j}}$ is the fuzzy state probability associated with the state $r_{i}, r_{i}=$ $0,1, \ldots, M_{i}$. As shown in (4), $\widetilde{p}_{i j, l_{j}}$ is a function of $\widetilde{\lambda}_{i j}(k, k-1)$ and $\tilde{\mu}_{i j}(k-1, k), k=1,2, \ldots, M_{i j}$, so the $\alpha$-cut of $\widetilde{p}_{i, r_{i}}$ can be obtained as

$$
\begin{aligned}
& \left(\tilde{p}_{i, r_{i}}\right)_{\alpha}=\left[\operatorname { m i n } _ { \Psi } \left\{\prod_{j=1}^{m_{i}} p_{i j, l_{j}} \mid \eta_{\tilde{\lambda}_{i j}}\left(\lambda_{i j}(k, k-1)\right)\right.\right. \\
& \left.\geq \alpha, \eta_{\tilde{\mu}_{i j}}\left(\mu_{i j}(k-1, k)\right) \geq \alpha\right\}, \\
& \max _{\Psi}\left\{\prod_{j=1}^{m_{i}} p_{i j, l_{j}} \mid \eta_{\tilde{\lambda}_{i j}}\left(\lambda_{i j}(k, k-1)\right)\right. \\
& \left.\left.\geq \alpha, \eta_{\tilde{\mu}_{i j}}\left(\mu_{i j}(k-1, k)\right) \geq \alpha\right\}\right]=\left[\left(p_{i, r_{i}}\right)_{\alpha}^{L},\right. \\
& \left.\left(p_{i, r_{i}}\right)_{\alpha}^{U}\right],
\end{aligned}
$$

where $\Psi=\left\{\lambda_{i j}(k, k-1) \in \Lambda_{i j}(k, k-1), \mu_{i j}(k-1, k) \in N_{i j}(k-\right.$ $\left.1, k) ; k=1, \ldots, M_{i j}\right\}$. The parametric programming to find the lower and upper bounds of the $\alpha$-cut of $\widetilde{p}_{i, r_{i}}$ is

$$
\begin{aligned}
\left(p_{i, r_{i}}\right)_{\alpha}^{L}=\min & \prod_{j=1}^{m_{i}} p_{i j, l_{j}}, \quad(0 \leq \alpha \leq 1) \\
\text { s.t. } \quad & \lambda_{i j}(k, k-1)_{\alpha}^{L} \leq \lambda_{i j}(k, k-1) \\
& \leq \lambda_{i j}(k, k-1)_{\alpha}^{U} \\
& \mu_{i j}(k-1, k)_{\alpha}^{L} \leq \mu_{i j}(k-1, k) \\
& \leq \mu_{i j}(k-1, k)_{\alpha}^{U} \\
& k=1,2, \ldots, M_{i j} ; j=1,2, \ldots, m_{i}, \\
\left(p_{i, r_{i}}\right)_{\alpha}^{U}=\max \quad & \prod_{j=1}^{m_{i}} p_{i j, l_{j}}, \quad(0 \leq \alpha \leq 1) \\
& \lambda_{i j}(k, k-1)_{\alpha}^{L} \leq \lambda_{i j}(k, k-1) \\
& \leq \lambda_{i j}(k, k-1)_{\alpha}^{U} \\
& \mu_{i j}(k-1, k)_{\alpha}^{L} \leq \mu_{i j}(k-1, k) \\
& \leq \mu_{i j}(k-1, k)_{\alpha}^{U} \\
& k=1,2, \ldots, M_{i j} ; j=1,2, \ldots, m_{i} .
\end{aligned}
$$

Let $\eta_{\tilde{g}_{i j, l_{j}}}\left(g_{i j, l_{j}}\right)$ denote the membership function of the fuzzy performance rate $\tilde{g}_{i j, l_{j}}$, and definition for the $\alpha$-cut of $\widetilde{g}_{i j, l_{j}}$ as crisp interval is as follows:

$$
\begin{aligned}
& \left(\tilde{g}_{i j, l_{j}}\right)_{\alpha}=\left[\min _{g_{i j, l_{j}} \in G_{i j, l_{j}}}\left\{g_{i j, l_{j}} \mid \eta_{\tilde{g}_{i j, l_{j}}}\left(g_{i j, l_{j}}\right) \geq \alpha\right\},\right. \\
& \left.\max _{g_{i j, l_{j}} \in G_{i j, l_{j}}}\left\{g_{i j, l_{j}} \mid \eta_{\tilde{g}_{i j, l_{j}}}\left(g_{i j, l_{j}}\right) \geq \alpha\right\}\right]=\left[\left(g_{i j, l_{j}}\right)_{\alpha}^{L},\right. \\
& \left.\left(g_{i j, l_{j}}\right)_{\alpha}^{U}\right],
\end{aligned}
$$

where $G_{i j, l_{j}}$ is the crisp set of the performance rate of the component $j$ of subsystem $i$.

According to Zadeh extension principle, the $\alpha$-cut of the fuzzy performance rate $\widetilde{g}_{i, r_{i}}=\sum_{j=1}^{m_{i}} \widetilde{g}_{i j, l_{j}}$ of the parallel subsystem $i$ can be obtained as

$$
\begin{aligned}
& \left(\widetilde{g}_{i, r_{i}}\right)_{\alpha}=\left[\operatorname { m i n } _ { \Upsilon } \left\{g_{i, r_{i}}=\sum_{j=1}^{m_{i}} g_{i j, l_{j}} \mid \eta_{\tilde{g}_{i j, l_{j}}}\left(g_{i j, l_{j}}\right) \geq \alpha, j\right.\right. \\
& \left.=1,2, \ldots, m_{i}\right\}, \max _{\Upsilon}\left\{g_{i, r_{i}}\right.
\end{aligned}
$$




$$
\begin{aligned}
& \left.\left.=\sum_{j=1}^{m_{i}} g_{i j, l_{j}} \mid \eta_{\tilde{g}_{i, l_{j}}}\left(g_{i j, l_{j}}\right) \geq \alpha, j=1,2, \ldots, m_{i}\right\}\right] \\
& =\left[\left(g_{i, r_{i}}\right)_{\alpha}^{L},\left(g_{i, r_{i}}\right)_{\alpha}^{U}\right],
\end{aligned}
$$

where $\Upsilon=\left\{g_{i j, l_{j}} \in G_{i j, l_{j}} ; j=1,2, \ldots, m_{i}\right\}$. The lower bound $\left(g_{i, r_{i}}\right)_{\alpha}^{L}$ and the upper bound $\left(g_{i, r_{i}}\right)_{\alpha}^{U}$ of the $\alpha$-cut of $\widetilde{g}_{i, r_{i}}$ can be obtained using the following parametric programming:

$$
\begin{aligned}
&\left(g_{i, r_{i}}\right)_{\alpha}^{L}=\min \sum_{j=1}^{m_{i}} g_{i j, l_{j}}, \quad(0 \leq \alpha \leq 1) \\
& \text { s.t. }\left(g_{i j, l_{j}}\right)_{\alpha}^{L} \leq g_{i j, l_{j}} \leq\left(g_{i j, l_{j}}\right)_{\alpha}^{U} \\
& j=1,2, \ldots, m_{i}, \\
&\left(g_{i, r_{i}}\right)_{\alpha}^{U}=\max \sum_{j=1}^{m_{i}} g_{i j, l_{j}}, \quad(0 \leq \alpha \leq 1) \\
& \quad\left(g_{i j, l_{j}}\right)_{\alpha}^{L} \leq g_{i j, l_{j}} \leq\left(g_{i j, l_{j}}\right)_{\alpha}^{U} \\
& j=1,2, \ldots, m_{i} .
\end{aligned}
$$

\section{Fuzzy Performance Rate and Fuzzy State Probability of the Entire System}

Using the fuzzy composition operator $\widetilde{\Theta}_{\phi_{S}}[25]$, we can obtain the fuzzy UGF of the RMSSPS, which is denote by $\widetilde{u}_{\mathrm{SP}}(z)$, based on the fuzzy UGF of its parallel subsystems

$$
\begin{aligned}
& \widetilde{u}_{\mathrm{SP}}(z)=\widetilde{\Theta}_{\phi_{S}}\left(\widetilde{u}_{1}(z), \widetilde{u}_{2}(z), \ldots, \widetilde{u}_{n}(z)\right) \\
& =\widetilde{\Theta}_{\phi_{S}}\left(\sum_{r_{1}=0}^{M_{1}} \widetilde{p}_{1, r_{1}} \cdot z^{\tilde{g}_{1, r_{1}}}, \sum_{r_{2}=0}^{M_{2}} \widetilde{p}_{2, r_{2}} \cdot z^{\widetilde{g}_{2, r_{2}}}, \ldots,\right. \\
& \left.\sum_{r_{n}=0}^{M_{n}} \widetilde{p}_{n, r_{n}} \cdot z^{\widetilde{g}_{n, r_{n}}}\right) \\
& =\sum_{r_{1}=0}^{M_{1}} \sum_{r_{2}=0}^{M_{2}} \cdots \sum_{r_{n}=0}^{M_{n}}\left(\prod_{i=1}^{n} \tilde{p}_{i, r_{i}} \cdot z^{\min \left\{\widetilde{g}_{1, r_{1}}, \widetilde{g}_{2, r_{2}}, \ldots, \tilde{g}_{n, r_{n}}\right\}}\right) \\
& =\sum_{l=0}^{M} \widetilde{p}_{l} \cdot z^{\widetilde{g}_{l}},
\end{aligned}
$$

where $M$ is the highest possible state for the entire system, $\widetilde{p}_{l}=\prod_{i=1}^{n} \widetilde{p}_{i, r_{i}}=\prod_{i=1}^{n} \prod_{j=1}^{m_{i}} \widetilde{p}_{i j, l_{j}}$ is the fuzzy probability of the system in the state $l$, and $\widetilde{g}_{l}=\min \left\{\widetilde{g}_{1, r_{1}}, \widetilde{g}_{2, r_{2}}, \ldots, \widetilde{g}_{n, r_{n}}\right\}=$ $\min \left\{\sum_{j=1}^{m_{1}} \widetilde{g}_{1 j, l_{j}}, \sum_{j=1}^{m_{2}} \widetilde{g}_{2 j, l_{j}}, \ldots, \sum_{j=1}^{m_{n}} \widetilde{g}_{n j, l_{j}}\right\}$ is the fuzzy performance rate of the system in state $l, l=0,1, \ldots, M$.
The $\alpha$-cut of $\widetilde{p}_{l}$ can be determined as

$$
\begin{aligned}
& \left(\tilde{p}_{l}\right)_{\alpha}=\left[\operatorname { m i n } _ { \Psi } \left\{\prod_{i=1}^{n} \prod_{j=1}^{m_{i}} p_{i j, l_{j}} \mid \eta_{\tilde{\lambda}_{i j}(k, k-1)}\left(\lambda_{i j}(k, k-1)\right)\right.\right. \\
& \left.\geq \alpha, \eta_{\widetilde{\mu}_{i j}(k-1, k)}\left(\mu_{i j}(k-1, k)\right) \geq \alpha\right\}, \\
& \max _{\Psi}\left\{\prod_{i=1}^{n} \prod_{j=1}^{m_{i}} p_{i j, l_{j}} \mid \eta_{\widetilde{\lambda}_{i j}(k, k-1)}\left(\lambda_{i j}(k, k-1)\right)\right. \\
& \left.\geq \alpha, \eta_{\widetilde{\mu}_{i j}(k-1, k)}\left(\mu_{i j}(k-1, k)\right) \geq \alpha\right\}=\left[\left(p_{l}\right)_{\alpha}^{L},\right. \\
& \left.\left(p_{l}\right)_{\alpha}^{U}\right],
\end{aligned}
$$

where $\Psi=\left\{\lambda_{i j}(k, k-1) \in \Lambda_{i j}(k, k-1), \mu_{i j}(k-1, k) \in N_{i j}(k-\right.$ $\left.1, k) ; k=1, \ldots, M_{i j}\right\}$. The parametric programming to find the lower and upper bounds of the $\alpha$-cut of $\widetilde{p}_{l}$ is

$$
\begin{aligned}
\left(p_{l}\right)_{\alpha}^{L}=\min \quad & \prod_{i=1}^{n} \prod_{j=1}^{m_{i}} p_{i j, l_{j}}, \quad(0 \leq \alpha \leq 1) \\
\text { s.t. } \quad & \lambda_{i j}(k, k-1)_{\alpha}^{L} \leq \lambda_{i j}(k, k-1) \\
& \leq \lambda_{i j}(k, k-1)_{\alpha}^{U} \\
& \mu_{i j}(k-1, k)_{\alpha}^{L} \leq \mu_{i j}(k-1, k) \\
& \leq \mu_{i j}(k-1, k)_{\alpha}^{U} \\
i=1,2, \ldots, n, j=1,2, \ldots, m_{i}, k=1, \ldots, M_{i j}, & (0 \leq \alpha \leq 1) \\
\left(p_{l}\right)_{\alpha}^{U}=\max \quad & \prod_{i=1}^{n} \prod_{j=1}^{m_{i}} p_{i j, l_{j}}, \quad\left(0 \leq 1, \ldots, M_{i j} .\right. \\
& \lambda_{i j}(k, k-1)_{\alpha}^{L} \leq \lambda_{i j}(k, k-1) \\
& \leq \lambda_{i j}(k, k-1)_{\alpha}^{U} \\
& \mu_{i j}(k-1, k)_{\alpha}^{L} \leq \mu_{i j}(k-1, k) \\
& \leq \mu_{i j}(k-1, k)_{\alpha}^{U} \\
i=1,2, \ldots, n, j=1,2, \ldots, m_{i}, k=1, k &
\end{aligned}
$$

The $\alpha$-cut of the fuzzy performance rate $\tilde{g}_{l}$ can be determined as

$$
\begin{aligned}
& \left(\tilde{g}_{l}\right)_{\alpha}=\left[\operatorname { m i n } _ { \Upsilon } \left\{\min \left\{\sum_{j=1}^{m_{1}} g_{1 j, l_{j}}, \ldots, \sum_{j=1}^{m_{n}} g_{n j, l_{j}}\right\} \mid\right.\right. \\
& \left.\eta_{\widetilde{g}_{i j, l_{j}}}\left(g_{i j, l_{j}}\right) \geq \alpha, i=1,2, \ldots, n, j=1,2, \ldots, m_{i}\right\},
\end{aligned}
$$




$$
\begin{aligned}
& \max _{\Upsilon}\left\{\min \left\{\sum_{j=1}^{m_{1}} g_{1 j, l_{j}}, \ldots, \sum_{j=1}^{m_{n}} g_{n j, l_{j}}\right\} \mid \eta_{\tilde{g}_{i j, l_{j}}}\left(g_{i j, l_{j}}\right)\right. \\
& \left.\left.\geq \alpha, i=1,2, \ldots, n, j=1,2, \ldots, m_{i}\right\}\right]=\left[\left(g_{l}\right)_{\alpha}^{L},\right. \\
& \left.\left(g_{l}\right)_{\alpha}^{U}\right],
\end{aligned}
$$

where $\Upsilon=\left\{g_{i j, l_{j}} \in G_{i j, l_{j}} ; j=1,2, \ldots, m_{i}\right\}$. The lower bound $(g)_{\alpha}^{L}$ and the upper bound $\left(g_{l}\right)_{\alpha}^{U}$ of the $\alpha$-cut of $\tilde{g}_{l}$ can be obtained using the following parametric programming:

$$
\begin{array}{cc}
\left(g_{l}\right)_{\alpha}^{L}=\min \quad\left\{\min \left\{\sum_{j=1}^{m_{1}} g_{1 j, l_{j}}, \sum_{j=1}^{m_{2}} g_{2 j, l_{j}}, \ldots, \sum_{j=1}^{m_{n}} g_{n j, l_{j}}\right\}\right\}, \\
\text { s.t. } \quad\left(g_{i j, l_{j}}\right)_{\alpha}^{L} \leq g_{i j, l_{j}} \leq\left(g_{i j, l_{j}}\right)_{\alpha}^{U} \quad \\
\left(g_{l}\right)_{\alpha}^{U}=\max \quad\left\{\min \left\{\sum_{j=1}^{m_{1}} g_{1 j, l_{j}}, \sum_{j=1}^{m_{2}} g_{2 j, l_{j}}, \ldots, \sum_{j=1}^{m_{n}} g_{n j, l_{j}}\right\}\right\}, \\
\text { s.t. } \quad\left(g_{i j, l_{j}}\right)_{\alpha}^{L} \leq g_{i j, l_{j}} \leq\left(g_{i j, l_{j}}\right)_{\alpha}^{U} \quad(0 \leq \alpha \leq 1) \\
i=1,2, \ldots, n, j=1,2, \ldots, m_{i} .
\end{array}
$$

\section{Fuzzy Steady-State Availability Assessment for the RMSSPS}

For the RMSSPS, the system availability is defined as the ability of the system to satisfy consumer demand. Here, the consumer demand is presented as fuzzy value $\widetilde{\omega}$, and the steady-state availability of the system is the fuzzy probability that the fuzzy performance rate of the system satisfies the fuzzy demand $\widetilde{\omega}$. So, we have

$$
\widetilde{A}(\widetilde{\omega})=\sum_{l=0}^{M} \widetilde{p}_{l} \cdot p\left(\widetilde{g}_{l} \geq \widetilde{\omega}\right),
$$

where $p\left(\widetilde{g}_{l} \geq \widetilde{\omega}\right)$ denote the possibility degree of $\widetilde{g}_{l} \geq \widetilde{\omega}$.

For crisp performance rate $g_{l}$ and consumer demand $\omega$, $p\left(g_{l} \geq \omega\right)=1$ if $g_{l} \geq \omega$, and $p\left(g_{l} \geq \omega\right)=0$ if $g_{l}<\omega$. In the fuzzy system model, the relationship between $\widetilde{g}_{l}$ and $\widetilde{\omega}$ is illustrated in Figure 3 under triangular fuzzy number. It can be seen from Figure 3 that $p\left(\widetilde{g}_{l} \geq \widetilde{\omega}_{1}\right)=0, p\left(\widetilde{g}_{l} \geq \widetilde{\omega}_{2}\right)=1$, and $0<p\left(\widetilde{g}_{l} \geq \widetilde{\omega}_{3(4)}\right)<1$. For $0<p\left(\widetilde{g}_{l} \geq \widetilde{\omega}_{3(4)}\right)<1$, we let $\widetilde{r}_{l}=\widetilde{g}_{l}-\widetilde{\omega}=\left\{r_{l}, \eta_{\widetilde{r}_{l}}\left(r_{l}\right) \mid r_{l}=g_{l}-\omega, g_{l} \in G_{l}, \omega \in\right.$ $W\}, \widetilde{r}_{l} \geq 0$ represents $\left\{r_{l}, \eta_{\widetilde{r}_{l}}\left(r_{l}\right) \mid r_{l} \geq 0, r_{l} \in R_{l}\right\}, \eta_{\widetilde{r}_{l}}\left(r_{l}\right)=$ $\sup _{\Omega} \min \left\{\eta_{\widetilde{g}_{l}}\left(g_{l}\right), \eta_{\widetilde{\omega}}(\omega) \mid r_{l}=g_{l}-\omega\right\}\left(\Omega=\left\{g_{l} \in G_{l}, \omega \in\right.\right.$ $W\})$ is the membership function of $\widetilde{r}_{l}, G_{l}, W$ and $R_{l}$ are the crisp universal sets of the system performance rate, consumer demand and $r_{l}$, respectively.

Based on the analysis above, we can replace $\widetilde{g}_{l} \geq \widetilde{\omega}$ with $\widetilde{r}_{l} \geq 0$; that is $p\left(\widetilde{g}_{l} \geq \widetilde{\omega}\right)=p\left(\widetilde{r}_{l} \geq 0\right)$. According to [27, 28], the possibility degree of $\widetilde{r}_{l} \geq 0$ is defined as

$$
p\left(\widetilde{r}_{l} \geq 0\right)=\frac{\int_{\substack{r_{l} \in R_{l} \\ r_{l} \geq 0}} \eta_{\tilde{r}_{\tilde{r}_{l}}}\left(r_{l}\right) d r_{l} \geq \alpha}{\int_{\substack{\left.\eta_{r_{l}} \in R_{l} \\ \eta_{r_{l}}\right) \geq \alpha}} \eta_{\widetilde{r}_{l}}\left(r_{l}\right) d r_{l}} .
$$

However, the definition of the possibility degree of $\widetilde{r}_{l} \geq 0$ in (21) is given by the membership function of $\widetilde{r}_{l}$. When the performance rates of the components and consumer demand are triangular fuzzy numbers, the membership function of $\widetilde{r}_{l}$ can be easily obtained. If system has a lot of components, and the performance rates of the components and consumer demand are arbitrary fuzzy representation, it is difficult to obtain the explicit expression of the membership function of $\widetilde{r}_{l}$, and some approximation techniques may be developed to calculate the membership function. Here, we tackle the problem by using the $\alpha$-cut approach. Based on the $\alpha$-cuts of $\tilde{g}_{l}$ and $\widetilde{\omega}$, the $\alpha$-cut of $\widetilde{r}_{l}$ can be easily determined. Let $\left[\left(r_{l}\right)_{\alpha}^{L},\left(r_{l}\right)_{\alpha}^{U}\right]$ denote the $\alpha$-cut of $\tilde{r}_{l}$; we define the possibility degree of $\widetilde{r}_{l} \geq 0$ as

$$
p\left(\widetilde{r}_{l} \geq 0\right)= \begin{cases}1, & \left(r_{l}\right)_{\alpha}^{L} \geq 0 \\ \frac{\left(r_{l}\right)_{\alpha}^{U}-0}{\left(r_{l}\right)_{\alpha}^{U}-\left(r_{l}\right)_{\alpha}^{L},} & \left(r_{l}\right)_{\alpha}^{L}<0, \quad\left(r_{l}\right)_{\alpha}^{U}>0 \\ 0, & \left(r_{l}\right)_{\alpha}^{U}<0 .\end{cases}
$$

Hence, the fuzzy steady-state availability of the system for a given fuzzy demand $\widetilde{\omega}$ can be rewritten as

$$
\widetilde{A}(\widetilde{\omega})=\sum_{l=0}^{M} \tilde{p}_{l} \cdot p\left(\widetilde{r}_{l} \geq 0\right) .
$$

We denote the $\alpha$-cut of $\widetilde{A}(\widetilde{\omega})$ by $(\widetilde{A}(\widetilde{\omega}))_{\alpha}=\left[(A(\omega))_{\alpha}^{L}\right.$, $\left.(A(\omega))_{\alpha}^{U}\right]$; the lower and upper bounds of $(\widetilde{A}(\widetilde{\omega}))_{\alpha}$ can be determined using the following parametric programming:

$$
\begin{aligned}
&(A(\omega))_{\alpha}^{L}=\min \sum_{l=0}^{M} p_{l} \cdot p\left(\widetilde{r}_{l} \geq 0\right), \quad(0 \leq \alpha \leq 1) \\
& \text { s.t. } \quad\left(p_{l}\right)_{\alpha}^{L} \leq p_{l} \leq\left(p_{l}\right)_{\alpha}^{U}, \\
& \quad l=0,1, \ldots, M, \\
& \sum_{l=0}^{M} p_{l}=1,
\end{aligned}
$$




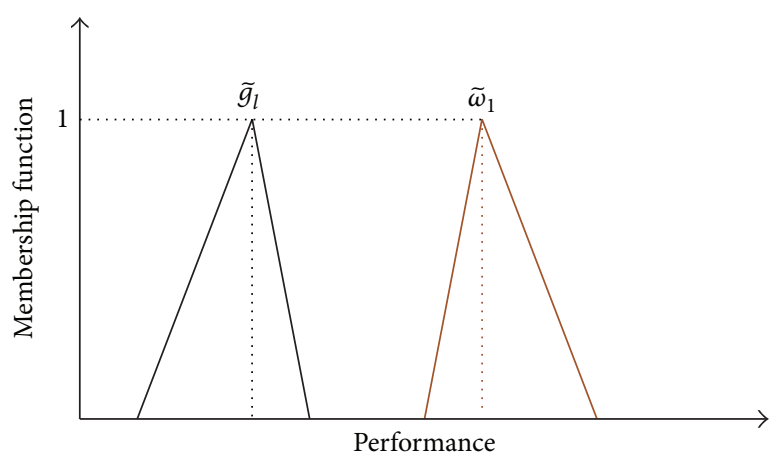

(a)

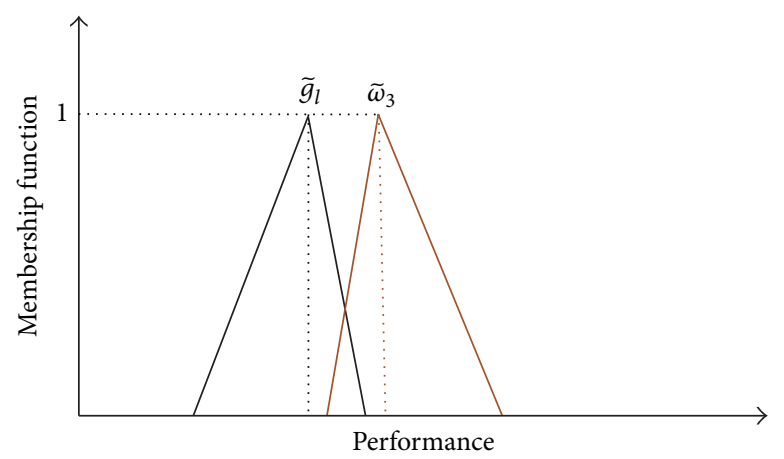

(c)

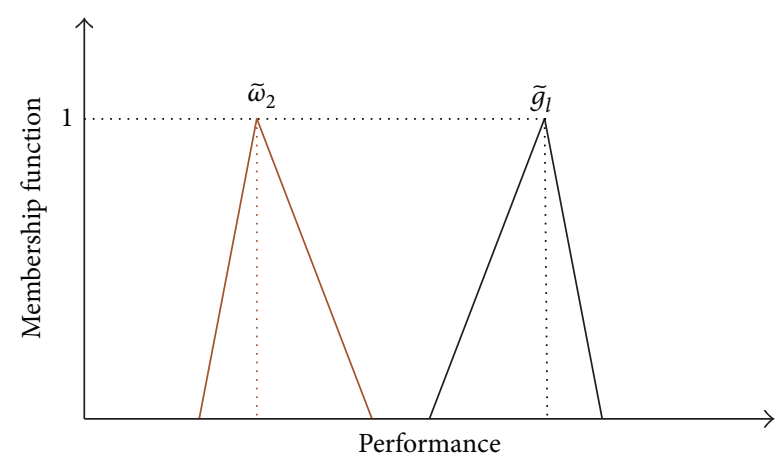

(b)

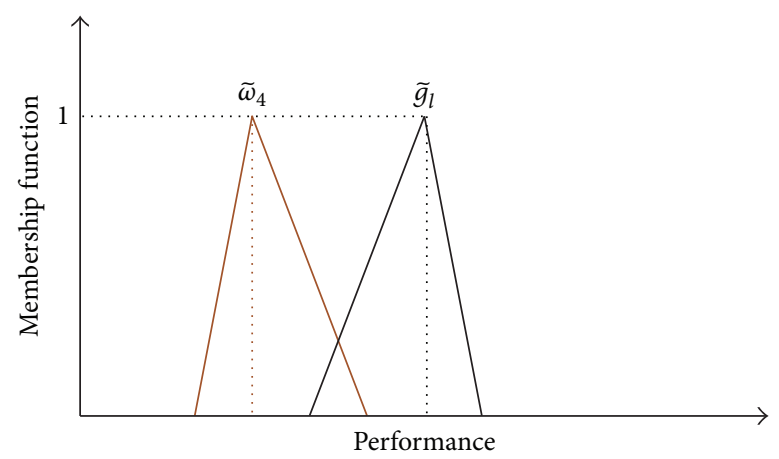

(d)

FIgURE 3: Fuzzy performance rate and fuzzy demand.

$$
\begin{aligned}
(A(\omega))_{\alpha}^{U}=\max & \sum_{l=0}^{M} p_{l} \cdot p\left(\widetilde{r}_{l} \geq 0\right), \quad(0 \leq \alpha \leq 1) \\
\text { s.t. } \quad & \left(p_{l}\right)_{\alpha}^{L} \leq p_{l} \leq\left(p_{l}\right)_{\alpha}^{U}, \\
& l=0,1, \ldots, M, \\
& \sum_{l=0}^{M} p_{l}=1 .
\end{aligned}
$$

In order to obtain rational outcome of the system steadystate availability, the equality constraint $\sum_{l=0}^{M} p_{l}=1$ is added into the parametric programming.

\section{Illustrative Example}

Consider a flow transmission multistate series-parallel system with three repairable components. In this example, the system consists of $n=2$ parallel subsystems connected in series, and subsystems 1 and 2 consist of $m_{1}=2$ and $m_{2}=1$ components, respectively. The parameters for the components are represented as triangular fuzzy values. The fuzzy transition rates and fuzzy performance rates of each component are presented in Tables 1 and 2, respectively. Suppose that the fuzzy demand $\widetilde{\omega}$ is a triangular fuzzy number $(1.9,2.1,2.3)$.
TABle 1: The fuzzy transition rates for the components of each subsystem.

\begin{tabular}{lccc}
\hline \multicolumn{2}{c}{ Subsystem 1} & \multicolumn{2}{c}{ Subsystem 2} \\
\hline$\widetilde{\lambda}_{11}(1,0)$ & $\tilde{\lambda}_{12}(1,0)$ & $\tilde{\lambda}_{21}(2,1)$ & $\tilde{\lambda}_{21}(1,0)$ \\
$(0.04,0.05$, & $(0.03,0.04$, & $(0.02,0.03$, & $(0.01,0.02$, \\
$0.06)$ & $0.05)$ & $0.04)$ & $0.03)$ \\
\hline$\tilde{\mu}_{11}(0,1)$ & $\tilde{\mu}_{12}(0,1)$ & $\tilde{\mu}_{21}(1,2)$ & $\tilde{\mu}_{21}(0,1)$ \\
$(0.4,0.5,0.6)$ & $(0.25,0.3,0.35)$ & $(0.2,0.3,0.4)$ & $(0.15,0.2,0.25)$ \\
\hline
\end{tabular}

TABLE 2: The fuzzy performance rates for the components of each subsystem.

\begin{tabular}{lccc}
\hline State & \multicolumn{2}{c}{ Subsystem 1 } & Subsystem 2 \\
\hline \multirow{2}{*}{$\widetilde{g}_{11,0}$} & $\widetilde{\mathfrak{g}}_{12,0}$ & $\widetilde{\mathfrak{g}}_{21,0}$ \\
& 0 & 0 & 0 \\
\hline 1 & $\widetilde{g}_{11,1}$ & $\widetilde{\mathfrak{g}}_{12,1}$ & $\widetilde{\mathfrak{g}}_{21,1}$ \\
& $(1.5,1.6,1.7)$ & $(1.8,2.0,2.2)$ & $(2.1,2.4,2.7)$ \\
\hline \multirow{2}{*}{2} & $\backslash$ & $\backslash$ & $\widetilde{\mathfrak{g}}_{21,2}$ \\
& $\backslash$ & $\backslash$ & $(3.6,3.7,3.8)$ \\
\hline
\end{tabular}

According to the method of determining the $\alpha$-cut of the triangular fuzzy number, the $\alpha$-cuts of the fuzzy transition rates for each component are

$$
\begin{aligned}
& {\left[\lambda_{11}(1,0)_{\alpha}^{L}, \lambda_{11}(1,0)_{\alpha}^{U}\right]} \\
& \quad=[0.04+0.01 \alpha, 0.06-0.01 \alpha],
\end{aligned}
$$




$$
\begin{array}{lcl}
{\left[\mu_{11}(0,1)_{\alpha}^{L}, \mu_{11}(0,1)_{\alpha}^{U}\right]=[0.4+0.1 \alpha, 0.6-0.1 \alpha],} & {\left[\mu_{21}(1,2)_{\alpha}^{L}, \mu_{21}(1,2)_{\alpha}^{U}\right]=[0.2+0.1 \alpha, 0.4-0.1 \alpha]} \\
{\left[\lambda_{12}(1,0)_{\alpha}^{L}, \lambda_{12}(1,0)_{\alpha}^{U}\right]} & {\left[\lambda_{21}(1,0)_{\alpha}^{L}, \lambda_{21}(1,0)_{\alpha}^{U}\right]} \\
=[0.03+0.01 \alpha, 0.05-0.01 \alpha], & =[0.01+0.01 \alpha, 0.03-0.01 \alpha] \\
{\left[\mu_{12}(0,1)_{\alpha}^{L}, \mu_{12}(0,1)_{\alpha}^{U}\right]} & {\left[\mu_{21}(0,1)_{\alpha}^{L}, \mu_{21}(0,1)_{\alpha}^{U}\right]} \\
=[0.25+0.05 \alpha, 0.35-0.05 \alpha], & =[0.15+0.05 \alpha, 0.25-0.05 \alpha]
\end{array}
$$$$
\left[\lambda_{21}(2,1)_{\alpha}^{L}, \lambda_{21}(2,1)_{\alpha}^{U}\right]
$$$$
=[0.02+0.01 \alpha, 0.04-0.01 \alpha] \text {, }
$$

In accordance with (4), we can obtain the fuzzy state probabilities of the components of subsystems 1 and 2

$$
\begin{aligned}
& \widetilde{p}_{11,0}=\frac{\tilde{\lambda}_{11}(1,0)}{\widetilde{\lambda}_{11}(1,0)+\widetilde{\mu}_{11}(0,1)} \\
& \widetilde{p}_{11,1}=\frac{\widetilde{\mu}_{11}(0,1)}{\widetilde{\lambda}_{11}(1,0)+\widetilde{\mu}_{11}(0,1)} \\
& \tilde{p}_{12,0}=\frac{\tilde{\lambda}_{12}(1,0)}{\widetilde{\lambda}_{12}(1,0)+\tilde{\mu}_{12}(0,1)} \\
& \widetilde{p}_{12,1}=\frac{\tilde{\mu}_{12}(0,1)}{\widetilde{\lambda}_{12}(1,0)+\tilde{\mu}_{12}(0,1)} \\
& \tilde{p}_{21,0}=\frac{1}{\left(1+\widetilde{\mu}_{21}(0,1) / \widetilde{\lambda}_{21}(1,0)+\widetilde{\mu}_{21}(0,1) \widetilde{\mu}_{21}(1,2) / \widetilde{\lambda}_{21}(1,0) \widetilde{\lambda}_{21}(2,1)\right)}, \\
& \widetilde{p}_{21,1}=\frac{\left(\tilde{\mu}_{21}(0,1) / \widetilde{\lambda}_{21}(1,0)\right)}{\left(1+\tilde{\mu}_{21}(0,1) / \widetilde{\lambda}_{21}(1,0)+\widetilde{\mu}_{21}(0,1) \tilde{\mu}_{21}(1,2) / \widetilde{\lambda}_{21}(1,0) \tilde{\lambda}_{21}(2,1)\right)}, \\
& \tilde{p}_{21,2}=\frac{\left(\tilde{\mu}_{21}(1,2) \tilde{\mu}_{21}(0,1) / \widetilde{\lambda}_{21}(2,1) \widetilde{\lambda}_{21}(1,0)\right)}{\left(1+\tilde{\mu}_{21}(0,1) / \widetilde{\lambda}_{21}(1,0)+\widetilde{\mu}_{21}(0,1) \widetilde{\mu}_{21}(1,2) / \widetilde{\lambda}_{21}(1,0) \widetilde{\lambda}_{21}(2,1)\right)} .
\end{aligned}
$$

The fuzzy UGFs of the components 1 and 2 in subsystem 1 can be described as follows:

$$
\begin{aligned}
& \tilde{u}_{11}(z)=\widetilde{p}_{11,0} \cdot z^{0}+\widetilde{p}_{11,1} \cdot z^{(1.5,1.6,1.7)} \\
& \tilde{u}_{12}(z)=\tilde{p}_{12,0} \cdot z^{0}+\tilde{p}_{12,1} \cdot z^{(1.8,2,2.2)}
\end{aligned}
$$

The fuzzy UGF of the component 1 in subsystem 2 can be written as

$$
\begin{aligned}
\tilde{u}_{21}(z)= & \tilde{p}_{21,0} \cdot z^{0}+\tilde{p}_{21,1} \cdot z^{(2.1,2.4,2.7)}+\widetilde{p}_{21,2} \\
& \cdot z^{(3.6,3.7,3.8)}
\end{aligned}
$$

Based on the individual fuzzy UGFs of the components 1 and 2 in subsystem 1 given above, we can get the fuzzy
UGF of the parallel subsystem 1 by using operator $\widetilde{\Theta}_{\phi_{P}}$ as follows:

$$
\begin{aligned}
\widetilde{u}_{1}(z)= & \widetilde{\Theta}_{\phi_{P}}\left(\widetilde{u}_{11}(z), \tilde{u}_{12}(z)\right) \\
= & \widetilde{p}_{11,0} \widetilde{p}_{12,0} \cdot z^{0}+\widetilde{p}_{11,1} \widetilde{p}_{12,0} \cdot z^{(1.5,1.6,1.7)} \\
& +\widetilde{p}_{11,0} \widetilde{p}_{12,1} \cdot z^{(1.8,2,2.2)}+\widetilde{p}_{11,1} \widetilde{p}_{12,1} \\
& \cdot z^{(3.3,3.6,3.9)} .
\end{aligned}
$$

The fuzzy UGF of subsystem 2 with one component is

$$
\begin{aligned}
\widetilde{u}_{2}(z)= & \widetilde{u}_{21}(z) \\
= & \widetilde{p}_{21,0} \cdot z^{0}+\widetilde{p}_{21,1} \cdot z^{(2.1,2.4,2.7)}+\widetilde{p}_{21,2} \\
& \cdot z^{(3.6,3.7,3.8)} .
\end{aligned}
$$


TABLE 3: The $\alpha$-cuts of the fuzzy state probabilities at $11 \alpha$ values.

\begin{tabular}{lccccc}
\hline$\alpha$ & {$\left[\left(p_{0}\right)_{\alpha}^{L},\left(p_{0}\right)_{\alpha}^{U}\right]$} & {$\left[\left(p_{1}\right)_{\alpha}^{L},\left(p_{1}\right)_{\alpha}^{U}\right]$} & {$\left[\left(p_{l}\right)_{\alpha}^{L},\left(p_{l}\right)_{\alpha}^{U}\right]$} & {$\left[\left(p_{2}\right)_{\alpha}^{L},\left(p_{2}\right)_{\alpha}^{U}\right]$} & {$\left[\left(p_{3}\right)_{\alpha}^{L},\left(p_{3}\right)_{\alpha}^{U}\right]$} \\
\hline 0.0 & {$[0.0068,0.0533]$} & {$[0.0664,0.1559]$} & {$[0.0504,0.1199]$} & {$[0.0342,0.1430]$} & {$\left[\left(p_{4}\right)_{\alpha}^{L},\left(p_{4}\right)_{\alpha}^{U}\right]$} \\
0.1 & {$[0.0076,0.0482]$} & {$[0.0700,0.1504]$} & {$[0.0530,0.1152]$} & {$[0.0371,0.1334]$} & {$[0.6008,0.8122]$} \\
0.2 & {$[0.0085,0.0436]$} & {$[0.0737,0.1449]$} & {$[0.0556,0.1106]$} & {$[0.0402,0.1246]$} & {$[0.6166,0.8034]$} \\
0.3 & {$[0.0095,0.0394]$} & {$[0.0774,0.1396]$} & {$[0.0583,0.1062]$} & {$[0.0435,0.1164]$} & {$[0.6317,0.7944]$} \\
0.4 & {$[0.0106,0.0357]$} & {$[0.0812,0.1345]$} & {$[0.0610,0.1020]$} & {$[0.0469,0.1088]$} & {$[0.6462,0.7850]$} \\
0.5 & {$[0.0117,0.0323]$} & {$[0.0851,0.1294]$} & {$[0.0639,0.0979]$} & {$[0.0506,0.1017]$} & {$[0.6602,0.7754]$} \\
0.6 & {$[0.0130,0.0292]$} & {$[0.0891,0.1245]$} & {$[0.0668,0.0939]$} & {$[0.0544,0.0950]$} & {$[0.6736,0.7655]$} \\
0.7 & {$[0.0145,0.0265]$} & {$[0.0932,0.1197]$} & {$[0.0698,0.0901]$} & {$[0.0585,0.0888]$} & {$[0.6865,0.7553]$} \\
0.8 & {$[0.0160,0.0240]$} & {$[0.0974,0.1150]$} & {$[0.0730,0.0865]$} & {$[0.0628,0.0829]$} & {$[0.6990,0.7448]$} \\
0.9 & {$[0.0177,0.0217]$} & {$[0.1016,0.1105]$} & {$[0.0762,0.0829]$} & {$[0.0674,0.0774]$} & {$[0.7110,0.7339]$} \\
1.0 & {$[0.0196,0.0196]$} & {$[0.1060,0.1060]$} & {$[0.0795,0.0795]$} & {$[0.0723,0.0723]$} & {$[0.7226,0.7226]$} \\
\hline
\end{tabular}

In order to find the fuzzy UGF of the entire system, the operator $\widetilde{\Theta}_{\phi_{S}}$ is applied to $\widetilde{u}_{1}(z)$ and $\widetilde{u}_{2}(z)$ based on the composition algorithm in Section 4. We have

$$
\begin{aligned}
& \widetilde{u}_{\mathrm{SP}}(z)=\widetilde{\Theta}_{\phi_{S}}\left(\widetilde{u}_{1}(z), \widetilde{u}_{2}(z)\right)=\left(\widetilde { p } _ { 2 1 , 0 } \left(\widetilde{p}_{11,0} \widetilde{p}_{12,0}\right.\right. \\
& \left.\quad+\widetilde{p}_{11,1} \widetilde{p}_{12,0}+\widetilde{p}_{11,0} \widetilde{p}_{12,1}+\widetilde{p}_{11,1} \widetilde{p}_{12,1}\right) \\
& \left.+\widetilde{p}_{11,0} \widetilde{p}_{12,0}\left(\widetilde{p}_{21,1}+\widetilde{p}_{21,2}\right)\right) \cdot z^{0}+\widetilde{p}_{11,1} \widetilde{p}_{12,0}\left(\widetilde{p}_{21,1}\right. \\
& \left.+\widetilde{p}_{21,2}\right) \cdot z^{(1.5,1.6,1.7)}+\widetilde{p}_{11,0} \widetilde{p}_{12,1}\left(\widetilde{p}_{21,1}+\widetilde{p}_{21,2}\right) \\
& \quad \cdot z^{(1.8,2,2.2)}+\widetilde{p}_{21,1} \widetilde{p}_{11,1} \widetilde{p}_{12,1} \cdot z^{(2.1,2.4,2.7)} \\
& \quad+\widetilde{p}_{21,2} \widetilde{p}_{11,1} \widetilde{p}_{12,1} \cdot z^{(3.3,3.6,3.8)}
\end{aligned}
$$

The fuzzy UGF of the entire system $\widetilde{u}_{\mathrm{SP}}(z)$ can be written as

$$
\widetilde{u}_{\mathrm{SP}}(z)=\sum_{l=0}^{4} \widetilde{p}_{l} \cdot z^{\widetilde{\mathcal{g}}_{l}}
$$

That is, the fuzzy performance rates of the system are

$$
\begin{aligned}
& \widetilde{g}_{0}=0, \\
& \widetilde{g}_{1}=(1.5,1.6,1.7), \\
& \widetilde{g}_{2}=(1.8,2,2.2), \\
& \widetilde{g}_{3}=(2.1,2.4,2.7), \\
& \widetilde{g}_{4}=(3.3,3.6,3.8) .
\end{aligned}
$$

The fuzzy probabilities corresponding to the fuzzy performance rates are

$$
\begin{aligned}
\widetilde{p}_{0}= & \widetilde{p}_{21,0} \widetilde{p}_{11,0} \widetilde{p}_{12,0}+\widetilde{p}_{21,0} \widetilde{p}_{11,1} \widetilde{p}_{12,0}+\widetilde{p}_{21,0} \widetilde{p}_{11,0} \widetilde{p}_{12,1} \\
& +\widetilde{p}_{21,0} \widetilde{p}_{11,1} \widetilde{p}_{12,1}+\widetilde{p}_{11,0} \widetilde{p}_{12,0} \widetilde{p}_{21,1} \\
& +\widetilde{p}_{11,0} \widetilde{p}_{12,0} \widetilde{p}_{21,2},
\end{aligned}
$$

$$
\begin{aligned}
& \tilde{p}_{1}=\widetilde{p}_{11,1} \widetilde{p}_{12,0} \widetilde{p}_{21,1}+\widetilde{p}_{11,1} \widetilde{p}_{12,0} \widetilde{p}_{21,2}, \\
& \widetilde{p}_{2}=\widetilde{p}_{11,0} \widetilde{p}_{12,1} \widetilde{p}_{21,1}+\widetilde{p}_{11,0} \widetilde{p}_{12,1} \widetilde{p}_{21,2}, \\
& \widetilde{p}_{3}=\widetilde{p}_{21,1} \widetilde{p}_{11,1} \widetilde{p}_{12,1}, \\
& \widetilde{p}_{4}=\widetilde{p}_{21,2} \widetilde{p}_{11,1} \widetilde{p}_{12,1} .
\end{aligned}
$$

The $\alpha$-cuts of $\widetilde{p}_{l}(l=0,1,2,3,4)$ can be obtained by (17). Due to the complicated form of the objective function, it is impossible to represent the optimal solution $\left(p_{l}\right)_{\alpha}^{L}$ and $\left(p_{l}\right)_{\alpha}^{U}$ in terms of $\alpha, l=0,1,2,3,4$. With the help of Mathematica program system, the $\alpha$-cuts of $\tilde{p}_{l}(l=0,1,2,3,4)$ can be computed at some distinct $\alpha$ values. The rough shapes of the membership functions for $\tilde{p}_{l}(l=0,1,2,3,4)$ can be determined according to different $\alpha$ values.

Table 3 presents the $\alpha$-cuts of $\widetilde{p}_{l}(l=0,1,2,3,4)$ at 11 distinct $\alpha$ values: $0.0,0.1, \ldots, 1.0$. Based on the results shown in Table 3, we can see that it is impossible for the values of $\widetilde{p}_{4}$ to fall below 0.5844 or exceed 0.8208 though the state probability is fuzzy, and the value of $\tilde{p}_{4}$ is equal to the result regarding transition rates and performance rates as crisp values when $\alpha=1$. Similarly, one can read the rest of the results for $\tilde{p}_{l}(l=0,1,2,3,4)$ presented in Table 3 . Figure 4 plots the rough shapes of the membership functions for $\tilde{p}_{l}(l=0,1,2,3,4)$ constructed from 51 values of $\alpha$ : $0,0.02,0.04, \ldots, 1.00$.

According to (22)-(24), the $\alpha$-cut of the fuzzy steady-state availability $\widetilde{A}(\widetilde{\omega})$ can be computed and the rough shape of the membership function for $\widetilde{A}(\widetilde{\omega})$ can be plotted at different $\alpha$ values. The $\alpha$-cut and the rough shape of the membership function for $\widetilde{A}(\widetilde{\omega})$ at different $\alpha$ values according to Liu's method [28] and our proposed method are presented in Table 4 and Figure 5, respectively.

It can be seen from Table 4 that the $\alpha$-cut of the fuzzy steady-state availability calculated by our proposed method is slightly different with that calculated by Liu's method [28] when $0.0 \leq \alpha<0.3$ and $0.6 \leq \alpha<0.8$, it is great when $0.3 \leq$ $\alpha<0.6$, and the results are the same when $0.8 \leq \alpha \leq 1.0$. The proposed method need not to solve the membership function 


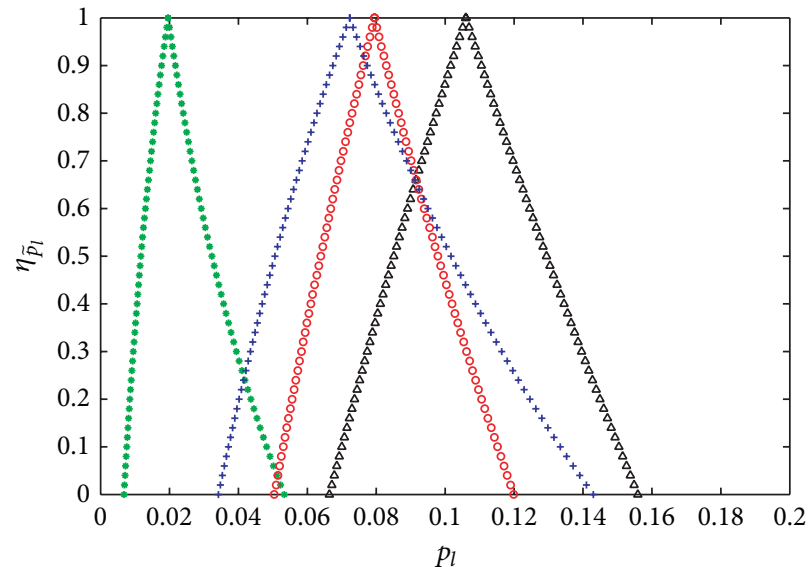

$$
\begin{array}{ll}
-l=0 \quad & \circ l=2
\end{array}
$$$$
\Delta l=1 \quad+l=3
$$

(a)

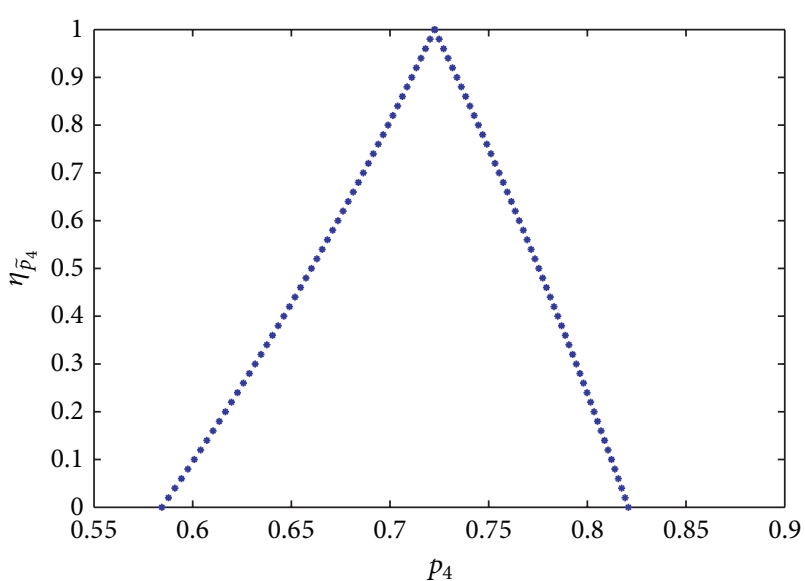

(b)

FIGURE 4: The membership functions for fuzzy state probabilities $\widetilde{p}_{l}(l=0,1,2,3,4)$.

TABLE 4: The $\alpha$-cut of the fuzzy steady-state availability $\widetilde{A}(\widetilde{\omega})$ at $11 \alpha$ values.

\begin{tabular}{lcc}
\hline$\alpha$ & $\begin{array}{c}\text { Liu's method } \\
{\left[(A(\omega))_{\alpha}^{L},(A(\omega))_{\alpha}^{U}\right]}\end{array}$ & $\begin{array}{c}\text { Proposed method } \\
{\left[(A(\omega))_{\alpha}^{L},(A(\omega))_{\alpha}^{U}\right]}\end{array}$ \\
\hline 0.0 & {$[0.6977,0.8861]$} & {$[0.6986,0.8842]$} \\
0.1 & {$[0.7119,0.8799]$} & {$[0.7136,0.8790]$} \\
0.2 & {$[0.7257,0.8737]$} & {$[0.7284,0.8740]$} \\
0.3 & {$[0.7392,0.8676]$} & {$[0.7430,0.8692]$} \\
0.4 & {$[0.7522,0.8618]$} & {$[0.7576,0.8650]$} \\
0.5 & {$[0.7608,0.8526]$} & {$[0.7649,0.8553]$} \\
0.6 & {$[0.7673,0.8417]$} & {$[0.7700,0.8436]$} \\
0.7 & {$[0.7701,0.8275]$} & {$[0.7712,0.8283]$} \\
0.8 & {$[0.7745,0.8136]$} & {$[0.7745,0.8136]$} \\
0.9 & {$[0.7849,0.8045]$} & {$[0.7849,0.8045]$} \\
1.0 & {$[0.7949,0.7949]$} & {$[0.7949,0.7949]$} \\
\hline
\end{tabular}

of $\widetilde{r}_{l}$ (see Section 5), and only need to determine the $\alpha$-cut of $\tilde{r}_{l}$. According to our proposed method, the shape of the membership function for the fuzzy steady-state availability $\widetilde{A}(\widetilde{\omega})$ is presented in Figure 5 compared with the one using the Liu's method [28].

\section{Conclusions}

This paper applies the concept of $\alpha$-cut and Zadeh's principle to a RMSSPS with fuzzy state transition rates and fuzzy performance rates, and then analyzes the system indices of interest. The fuzzy UGF technique works efficiently for the computation of the fuzzy state probabilities and fuzzy performance rates of the system. The parametric programming formulas are presented to find the $\alpha$-cuts of the fuzzy state probabilities, the fuzzy performance rates, and

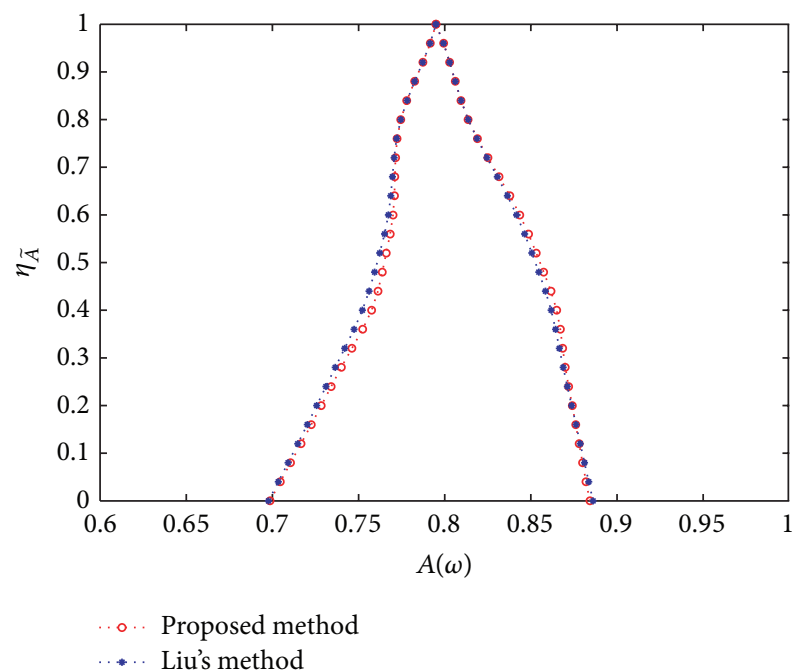

FIGURE 5: The membership function for fuzzy steady-state availability $\widetilde{A}(\widetilde{\omega})$.

the fuzzy steady-state availability of the entire system. A special availability evaluation approach is introduced when the system performance rates and the consumer demand are fuzzy values. Numerical technique method is used to determine the membership functions of the fuzzy state probabilities and the fuzzy availability for the entire system. An illustrative example is provided to show the validity of the proposed method. In future work, we will concern the development of fuzzy optimal design for the RMSSPS.

\section{Conflict of Interests}

The authors declare that there is no conflict of interests regarding the publication of this paper. 


\section{Acknowledgments}

This research work is supported in part by the Natural Science Foundation of Hebei Province (no. A2014203096 and G2012203136), the National Natural Science Foundation of China (no. 11201408), the Science Research Project of Yanshan University (no. 13LGA017), and Key Project of Natural Research of University of Hebei Province (no. ZH2012021). The authors are grateful to the editor and the reviewers for their insightful comments and suggestions.

\section{References}

[1] I. Ushakov, "Universal generating function," Soviet Journal of Computer and System Sciences, vol. 24, no. 5, pp. 118-129, 1986.

[2] A. Lisnianski and G. Levitin, Multi-State System Reliability Assessment, Optimization, Applications, World Scientific Publishers, Singapore, 2003.

[3] G. Levitin, Universal Generating Function and Its Application, Springer, Berlin, Germany, 2005.

[4] Z. Tian, G. Levitin, and M. J. Zuo, "A joint reliabilityredundancy optimization approach for multi-state seriesparallel systems," Reliability Engineering and System Safety, vol. 94, no. 10, pp. 1568-1576, 2009.

[5] T. Onisawa, "An application of fuzzy concepts to modelling of reliability analysis," Fuzzy Sets and Systems, vol. 37, no. 3, pp. 267-286, 1990.

[6] K. Y. Cai, C. Y. Wen, and M. L. Zhang, "Fuzzy variables as a basis for a theory of fuzzy reliability in the possibility context," Fuzzy Sets and Systems, vol. 42, no. 2, pp. 145-172, 1991.

[7] H.-Z. Huang, "Reliability analysis method in the presence of fuzziness attached to operating time," Microelectronics Reliability, vol. 35, no. 12, pp. 1483-1487, 1995.

[8] L. V. Utkin and S. V. Gurov, "A general formal approach for fuzzy reliability analysis in the possibility context," Fuzzy Sets and Systems, vol. 83, no. 2, pp. 203-213, 1996.

[9] B. Liu, "Uncertain risk analysis and uncertain reliability analysis," Journal of Uncertain Systems, vol. 4, no. 3, pp. 163-170, 2010.

[10] L. A. Zadeh, "Fuzzy sets," Information and Computation, vol. 8, no. 3, pp. 338-353, 1965.

[11] E. Munoz and E. H. Ruspini, "Simulation of fuzzy queueing systems with a variable number of servers, arrival rate, and service rate," IEEE Transactions on Fuzzy Systems, vol. 22, no. 4, pp. 892-903, 2014.

[12] J. K. Hamidi, K. Shahriar, B. Rezai, and H. Bejari, "Application of fuzzy set theory to rock engineering classification systems: an illustration of the rock mass excavability index," Rock Mechanics and Rock Engineering, vol. 43, no. 3, pp. 335-350, 2010.

[13] S. Bray, L. Caggiani, M. Dell'Orco, and M. Ottomanelli, "Measuring transport systems efficiency under uncertainty by fuzzy sets theory based data envelopment analysis," Procedia-Social and Behavioral Sciences, vol. 111, pp. 770-779, 2014.

[14] D. Giena, S. Jacqmarta, A. Sekloulia, and M. Baradb, "An approach based on fuzzy sets for manufacturing system design," International Journal of Production Research, vol. 41, no. 2, pp. 315-335, 2003.

[15] D. Petrovic, Y. Xie, K. Burnham, and R. Petrovic, "Coordinated control of distribution supply chains in the presence of fuzzy customer demand," European Journal of Operational Research, vol. 185, no. 1, pp. 146-158, 2008.
[16] R.-H. Liang and J.-H. Liao, "A fuzzy-optimization approach for generation scheduling with wind and solar energy systems," IEEE Transactions on Power Systems, vol. 22, no. 4, pp. 1665$1674,2007$.

[17] G. S. Mahapatra and T. K. Roy, "Fuzzy multi-objective mathematical programming on reliability optimization model," Applied Mathematics and Computation, vol. 174, no. 1, pp. 643659, 2006.

[18] L. Wang, Y. Qin, J. Xu, and L. Jia, "A fuzzy optimization model for high-speed railway timetable rescheduling," Discrete Dynamics in Nature and Society, vol. 2012, Article ID 827073, 22 pages, 2012.

[19] B. K. Wong and V. S. Lai, "A survey of the application of fuzzy set theory in production and operations management: 1998-2009," International Journal of Production Economics, vol. 129, no. 1, pp. 157-168, 2011.

[20] B. Praba, R. Sujatha, and S. Srikrishna, "Fuzzy reliability of non homogeneous unified fuzzy possibilistic Markov model," European Journal of Scientific Research, vol. 52, no. 4, pp. 495506, 2011.

[21] Y. Liu, X. Li, and J. Li, "Reliability analysis of random fuzzy unrepairable systems," Discrete Dynamics in Nature and Society, vol. 2014, Article ID 625985, 15 pages, 2014.

[22] M. Jain, S. C. Agrawal, and C. Preeti, "Fuzzy reliability evaluation of a repairable system with imperfect coverage, reboot and common-cause shock failure," International Journal of Engineering, vol. 25, no. 3, pp. 231-238, 2012.

[23] S. Wang and J. Watada, "Modelling redundancy allocation for a fuzzy random parallel-series system," Journal of Computational and Applied Mathematics, vol. 232, no. 2, pp. 539-557, 2009.

[24] K. Cai, Introduction to Fuzzy Reliability, Kluwer Academic Publishers, Dordrecht, The Netherlands, 1996.

[25] Y. Ding and A. Lisnianski, "Fuzzy universal generating functions for multi-state system reliability assessment," Fuzzy Sets and Systems, vol. 159, no. 3, pp. 307-324, 2008.

[26] Y. Ding, M. J. Zuo, A. Lisnianski, and Z. Tian, "Fuzzy multistate systems: general definitions, and performance assessment," IEEE Transactions on Reliability, vol. 57, no. 4, pp. 589-594, 2008.

[27] Y. Liu, H. Huang, and G. Levitin, "Reliability and performance assessment for fuzzy multi-state elements," Journal of Risk and Reliability, vol. 222, no. 4, pp. 675-686, 2008.

[28] Y. Liu and H.-Z. Huang, "Reliability assessment for fuzzy multi-state systems," International Journal of Systems Science. Principles and Applications of Systems and Integration, vol. 41, no. 4, pp. 365-379, 2010.

[29] W. Bamrungsetthapong and A. Pongpullponsak, "Parameter interval estimation of system reliability for repairable multistate series-parallel system with fuzzy data," The Scientific World Journal, vol. 2014, Article ID 275374, 10 pages, 2014.

[30] E. E. Lewis, Introduction to Reliability Engineering, John Wiley \& Sons, New York, NY, USA, 1987.

[31] L. A. Zadeh, "Fuzzy sets as a basis for a theory of possibility," Fuzzy Sets and Systems, vol. 1, no. 1, pp. 3-28, 1978.

[32] J.-C. Ke, H.-I. Huang, and C.-H. Lin, "Fuzzy analysis for steadystate availability: a mathematical programming approach," Engineering Optimization, vol. 38, no. 8, pp. 909-921, 2006.

[33] J.-C. Ke, H.-I. Huang, and C.-H. Lin, "Analysis on a queue system with heterogeneous servers and uncertain patterns," Journal of Industrial and Management Optimization, vol. 6, no. 1, pp. 57-71, 2010. 


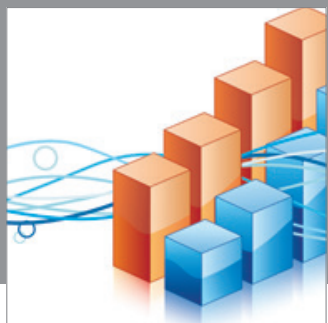

Advances in

Operations Research

mansans

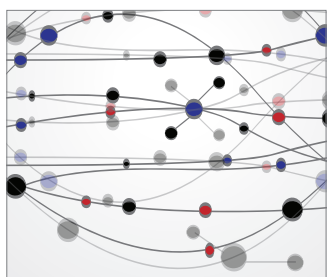

The Scientific World Journal
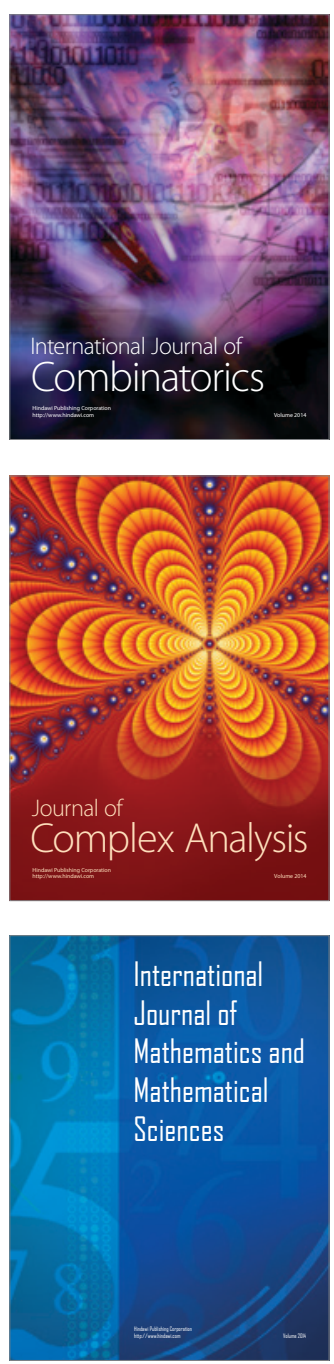
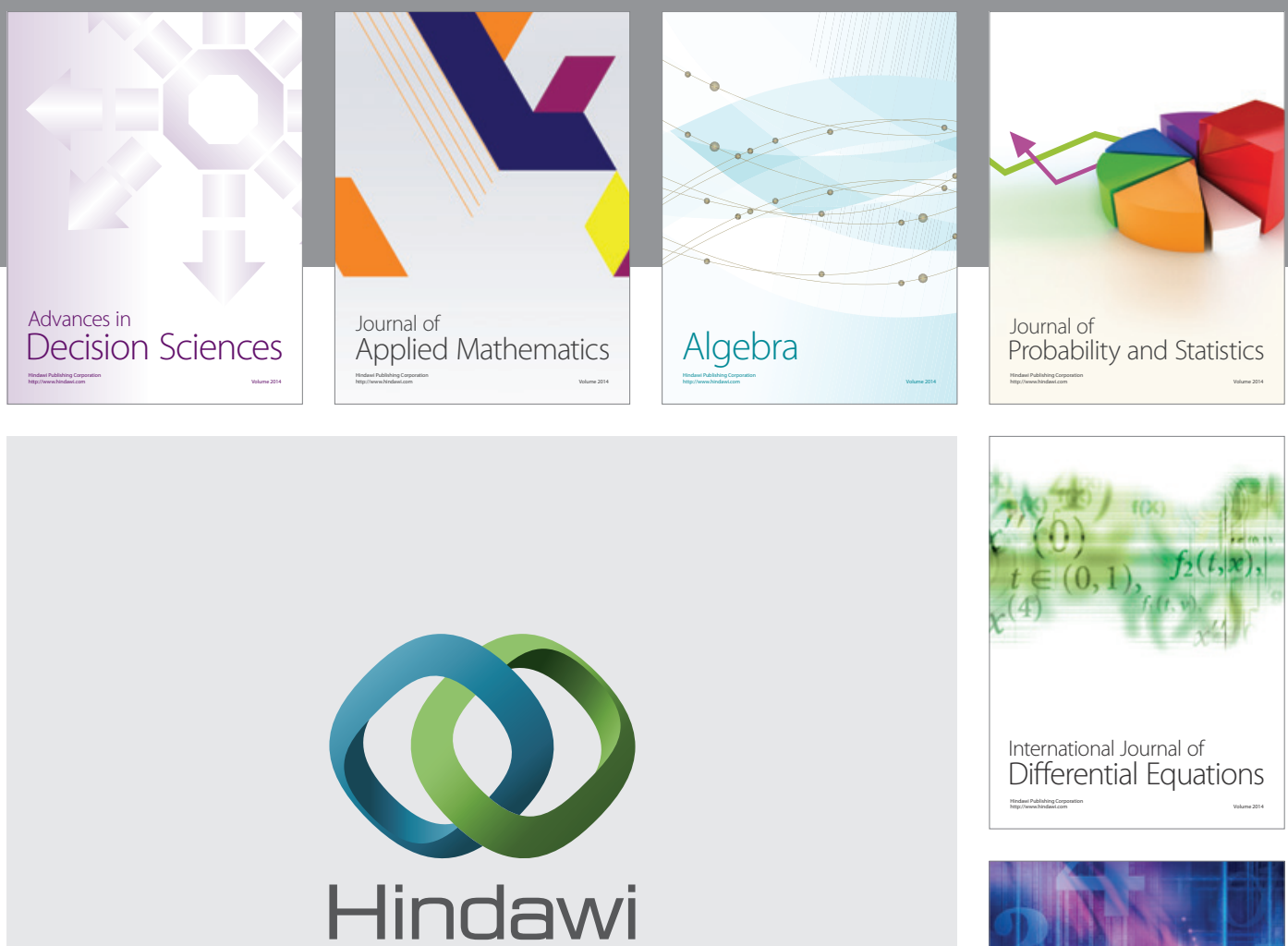

Submit your manuscripts at http://www.hindawi.com
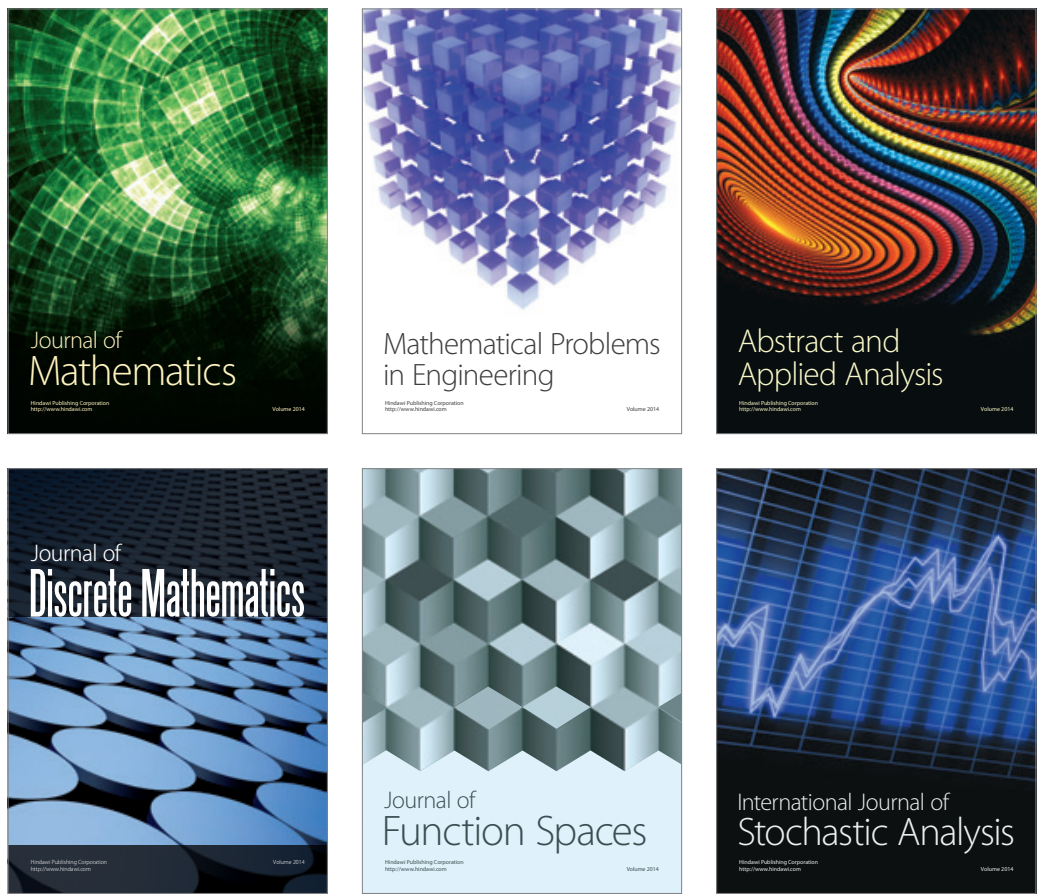

Journal of

Function Spaces

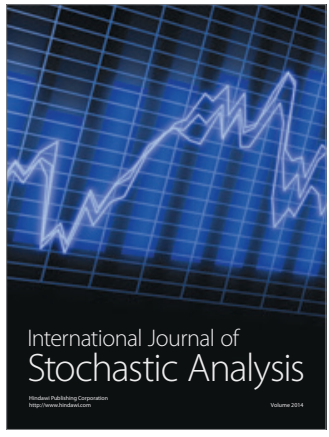

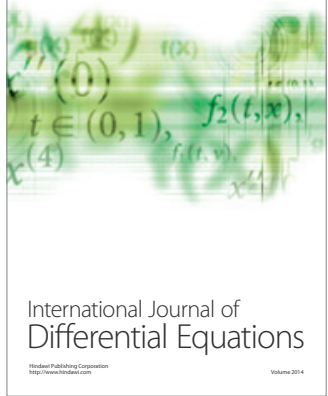
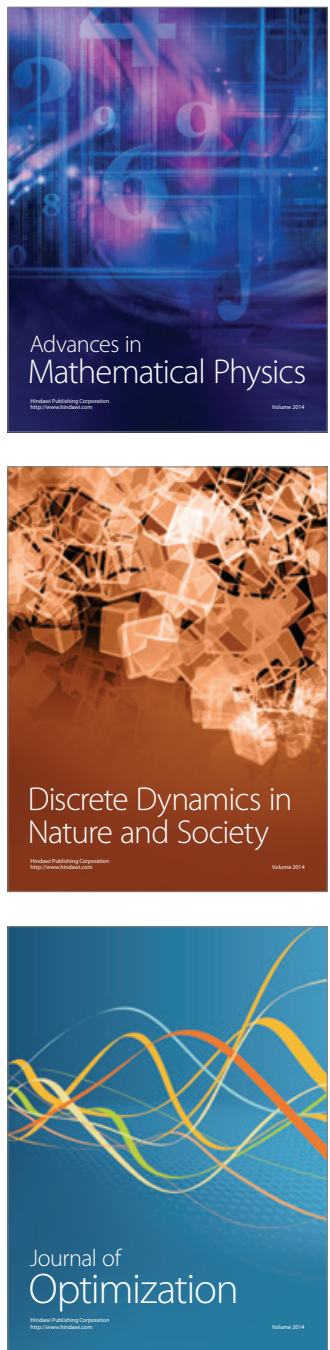